\title{
Modulation of hepatitis B surface antigen secretion by annexin II expressed in hepatitis B virus-producing hepatoma cells
}

\author{
YAMING LIU and PUJUN GAO \\ Department of Hepatology, First Hospital of Jilin University, Changchun, Jilin 130021, P.R. China
}

Received December 14, 2013; Accepted August 1, 2014

DOI: $10.3892 / \mathrm{mmr} .2014 .2602$

\begin{abstract}
The role of annexin II in hepatitis B virus (HBV)-associated hepatocellular carcinoma (HCC) remains to be elucidated. Intracellular hepatitis B surface antigen (HBsAg)-retention contributes to the induction of hepatocarcinogenesis. The present study aimed to investigate the regulation of $\mathrm{HBsAg}$ secretion by annexin II expressed in HBV-producing hepatoma cells. The expression of annexin II was analyzed using western blot analysis in SMMC-7721, HepG2, HepG2.2.15, 293T and Chinese hamster ovary (CHO) cells. $\mathrm{CHO}$ cells transfected with an annexin II plasmid were used as a positive control. The localization of annexin II and HBsAg was observed in the HepG2 and HepG2.2.15 cells using indirect immunofluorescence. HepG2.2.15 cells were transfected with a human immunodeficiency virus-type 1 viral infectivity factor-hemagglutinin (Vif-HA) plasmid or a control vector and, $24 \mathrm{~h}$ post-transfection, MG132 was added to the Vif-complemented HepG2.2.15 cells. Western blot analysis was performed to detect the expression of annexin II and Vif-HA. HepG2 cells were cotransfected with HBV and annexin II expression vectors. Western blot analysis was performed to examine the expression of annexin II and an Abbott chemiluminescence immunoassay was used to assess the levels of HBsAg. The expression of annexin II was lower in the HepG2.2.15 cells compared with the SMMC-7721 and HepG2 cells and the fluorescence signal of annexin II in the HepG2 cells was brighter than in the HepG2.2.15 cells. Annexin II colocalized with HBsAg in the cytosol of the HepG2.2.15 cells. MG132 was not able to increase the stability of annexin II expression in HepG2.2.15 cells. Annexin II reduced the secretion of HBsAg when compared with the control-transfected HepG2 cells. In conclusion, HBV downregulated the expression of annexin II and annexin II decreased the secretion of HBsAg in HBV-producing hepatoma cells in favor of intracellular HBsAg storage.
\end{abstract}

Correspondence to: Dr Pujun Gao, Department of Hepatology, First Hospital of Jilin University, 71 Xinmin Street, Changchun, Jilin 130021, P.R. China

E-mail: pujun_gao@163.com

Key words: annexin II, hepatitis B surface antigen, hepatitis B virus, hepatocyte

\section{Introduction}

Human hepatitis B virus (HBV) infection is a worldwide problem. More than $400,000,000$ individuals are chronically infected with HBV and $>1,000,000$ succumb to HBV-associated liver cirrhosis and hepatocellular carcinoma (HCC) (1). Chronic HBV infection is recognized as a major risk factor for $\mathrm{HCC}$ and the morbidity and mortality rates of HBV-associated HCC are high. However, there is no effective treatment for HCC.It is generally accepted that the pathogenesis of HBV-associated HCC is a multifactorial and synergistical process, which is mediated by immune responses (2). Certain studies have reported that the hepatitis B surface antigen (HBsAg) accumulates in hepatocytes and the truncated form of the large HBV envelope protein can lead to hepatocarcinogenesis $(3,4)$. In addition, HBV envelope proteins contain large, middle and small surface proteins. Viral replication and maturation occur in the endoplasmic reticulum (ER). HBV surface proteins accumulate in the ER of hepatocytes and initiate ER stress, which induces oxidative DNA damage and genomic instability (5). However, the mechanism underlying the accumulation of large quantities of viral envelope proteins in hepatocytes remains to be elucidated.

The $36 \mathrm{kDa}$ calcium-dependent phospholipid-binding protein, annexin II is present on the surface of the majority of eukaryotic cells and is involved in a number of biological processes (6). Annexin II is highly expressed in several types of human neoplasms, including pancreatic (7), gastric (8) and colorectal cancer (9). However, the expression of annexin II is low in prostate (10) and head and neck squamous cell carcinoma (11). At present, the expression of annexin II in liver cancer remains to be elucidated. The mRNA and protein levels of annexin II are increased in HCC tissues $(12,13)$, but are downregulated in HepG2.2.15 cells compared with HepG2 cells (14). It has been suggested that expression of the HBV genome inhibits the expression of annexin II by DNA methylation in the promoter of HepG2.2.15 cells (14). However, annexin II is a membrane-binding protein and the ubiquitin-proteasome system may target membrane-anchored or secretory pathway-compartmentalized proteins and cause protein degradation $(15,16)$. Therefore, further investigation is required to clarify the expression of annexin II in HBV-producing hepatoma cells, determine the factors affecting the expression of annexin II and investigate the possible function of annexin II in HBV-associated HCC. 
The present study aimed to detect the expression and localization of annexin II in different cell lines, clarify whether ubiquitination and the proteasome mediate the degradation of annexin II in HepG2.2.15 cells and investigate whether annexin II is important in the secretion, rather than the intracellular storage of HBsAg. The findings of the present study revealed the association between the expression of annexin II and the secretion of HBsAg in HBV infection.

\section{Materials and methods}

Plasmid. Annexin II was amplified by polymerase chain reaction $(\mathrm{PCR})$ using $\mathrm{pEGFP}$-annexin II, which was provided by Dr Carl E. Creuz (17). The following primers were used: forward 5'-CGGGATCCACCATGTCTACTGTTCACG-3' containing $\mathrm{BamHI}$ and reverse 5'-AAATATGCGGCCG CTCAGTCATCTCCACCA-3' containing NotI. The PCR products were cloned into pcDNA3.1(+) to generate a pcDNA3.1-annexin II expression vector and the inserted fragment was sequenced by Shanghai Generay Biotech Co., Ltd. (Shanghai, China).

Cell lines, culture and transfection. The HepG2.2.15 and Chinese hamster ovary $(\mathrm{CHO})-\mathrm{K} 1$ cell lines were obtained from the Academy of Military Medical Sciences (Beijing, China). The human embryonic kidney cell line 293T was obtained from the Institute of Virology and AIDS Research, First Hospital of Jilin University (Changchun, China). The human HCC cell lines HepG2 and SMMC-7721 were preserved in the Hepatology Institute, First Hospital of Jilin University (Changchun, China). These cells were cultured in Dulbecco's modified Eagle's medium (HyClone, Logan, UT, USA) supplemented with $10 \%$ fetal bovine serum (Gibco-BRL, Carlsbad, CA, USA), $100 \mathrm{U} / \mathrm{ml}$ penicillin and $100 \mu \mathrm{g} / \mathrm{ml}$ streptomycin (North China Pharmaceutical Group Corp., Shijiazhuang, China) at $37^{\circ} \mathrm{C}$ in a $5 \% \mathrm{CO}_{2}$ atmosphere. The HepG2.2.15 cells were cultured with $380 \mu \mathrm{g} / \mathrm{ml}$ geneticin G418 sulfate (Gibco-BRL). Transient transfections were performed using Lipofectamine 2000 transfection reagent (Invitrogen Life Technologies, Carlsbad, CA, USA) according to the manufacturers' instructions.

Antibodies. The mouse anti-annexin II monoclonal antibody (cat no. 610069) was purchased from BD Biosciences (San Jose, CA, USA). The mouse anti-HBsAg monoclonal antibody (cat no. BM0064) was obtained from Wuhan Boster Biological Technology, Ltd. (Wuhan, China), while the mouse anti-hemagglutinin (HA) monoclonal antibody (cat. no. A01244) and mouse anti- $\beta$-actin monoclonal antibody (cat no. A00702) were obtained from GenScript USA, Inc. (Piscataway, NJ, USA). Alkaline phosphatase-conjugated anti-mouse and anti-rabbit polyclonal secondary antibodies were obtained from Jackson ImmunoResearch, Inc. (West Grove, PA, USA). Fluorescein-conjugated anti-mouse and anti-rabbit polyclonal secondary antibodies were obtained from Invitrogen Life Technologies.

Western blot analysis. Cells were lysed in radioimmunoprecipitation assay buffer $(50 \mathrm{mM}$ Tris, $150 \mathrm{mM} \mathrm{NaCl}$, $1 \%$ NP-40, $0.5 \%$ sodium deoxycholate and 9 mM EDTA;
$\mathrm{pH}$ 7.4). The samples were mixed with $1 \mathrm{X}$ loading buffer $[1 \mathrm{M}$ Tris-HCl; $\mathrm{pH}$ 6.8, with $2.0 \%$ sodium dodecyl sulfate (SDS), $10 \%$ glycerol, $0.1 \mathrm{M}$ dithiothreitol and $0.2 \%$ bromphenol blue] and boiled for $20 \mathrm{~min}$, following which the proteins were separated by SDS-PAGE (Bio-Rad Laboratories, Inc., Hercules, CA, USA). The separated proteins on the gels were electrophoretically transferred onto a nitrocellulose membrane (Whatman, Piscataway, NJ, USA) at $20 \mathrm{~V}$ for $20 \mathrm{~min}$. The blotted membrane was probed with anti-annexin II, anti-HA, or anti- $\beta$-actin primary antibodies. The secondary antibodies used were alkaline phosphatase-conjugated anti-mouse or anti-rabbit immunoglobulin $\mathrm{G}$ and staining was performed using 5-bromo-4-chloro-3-indolyl phosphate and nitro blue tetrazolium solutions (Sigma-Aldrich, St. Louis, MO, USA).

Immunofluorescence. The cells cultured on coverslips were briefly rinsed with phosphate-buffered saline (PBS) solution and fixed using 4\% paraformaldehyde (Beijing Chemical Works, Beijing, China) for 20-30 min. The cells were rinsed, inhibited with $10 \%$ bovine serum albumin (Boster Biological Technology, Ltd., Wuhan, China) in PBS for $30 \mathrm{~min}$ at room temperature and incubated overnight at $4^{\circ} \mathrm{C}$ with primary antibodies diluted $1: 100$ in PBS plus $5 \%$ bovine serum albumin and $0.1 \%$ Triton X-100 (Amresco LLC, Solon, OH, USA). The cells were subsequently washed with PBS three times and incubated with secondary antibodies diluted 1:100 for $1 \mathrm{~h}$. The stained cells were observed using a FluoView FV1000 laser scanning confocal microscope (Olympus, Tokyo, Japan) equipped with an argon/krypton mixed gas laser (excitation 488 and $543 \mathrm{~nm}$ ). The blue (Hoechst-stained DNA), red (fluorescein isothiocyanate-stained HBsAg protein) and green (fluorescein isothiocyanate-stained annexin II protein) fluorescences were detected. A series of confocal images were captured using a UPLSAPO (magnification, x20, numeric aperture 0.75; Olympus). Each image was recorded using three-channel scanning and captured at an accuracy of 12 bits/pixel, as previously described $(17,18)$.

Assays for the expression of proteasome inhibitor-mediated annexin II in HepG2.2.15 cells. The HepG2.2.15 cells were transfected with either the human immunodeficiency virus-type 1 (HIV-1) viral infectivity factor-hemagglutinin (Vif-HA) plasmid, a control vector (VR1012) or the pEGFP-N1 plasmid. Subsequently, $24 \mathrm{~h}$ after transfection, the proteasome inhibitor MG132 was added to one of the Vif-complemented HepG2.2.15 cells. The cells were treated with MG132 for $16 \mathrm{~h}$ and all transfected cells were then lysed with lysis buffer (50 mM Tris, $150 \mathrm{mM} \mathrm{NaCl}, 1 \% \mathrm{NP}-40,0.5 \%$ sodium deoxycholate and 9 mM EDTA; $\mathrm{pH}$ 7.4) and measured by performing western blot analysis with anti-annexin II, anti-HA and anti- $\beta$-actin antibodies to determine the expression of annexin II, Vif and $\beta$-actin. The fluorescence signal in the GFP-complemented HepG2.2.15 cells was observed using an Olympus fluorescence microscope (Olympus).

Assays for HBsAg and annexin II from the cotransfected HepG2 cells. HepG2 cells were cotransfected with the HBV expression vector and annexin II plasmid or a control vector. Cells were harvested $48 \mathrm{~h}$ post-transfection, washed with PBS and suspended in lysis buffer (50 mM Tris- $\mathrm{HCl}$, 
A

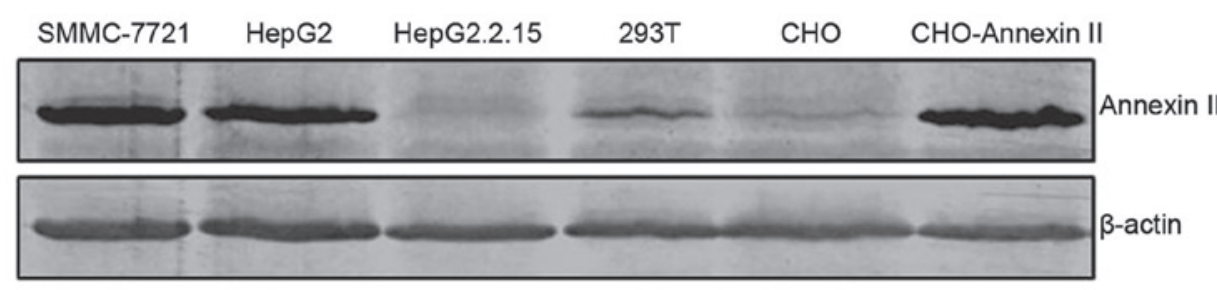

B
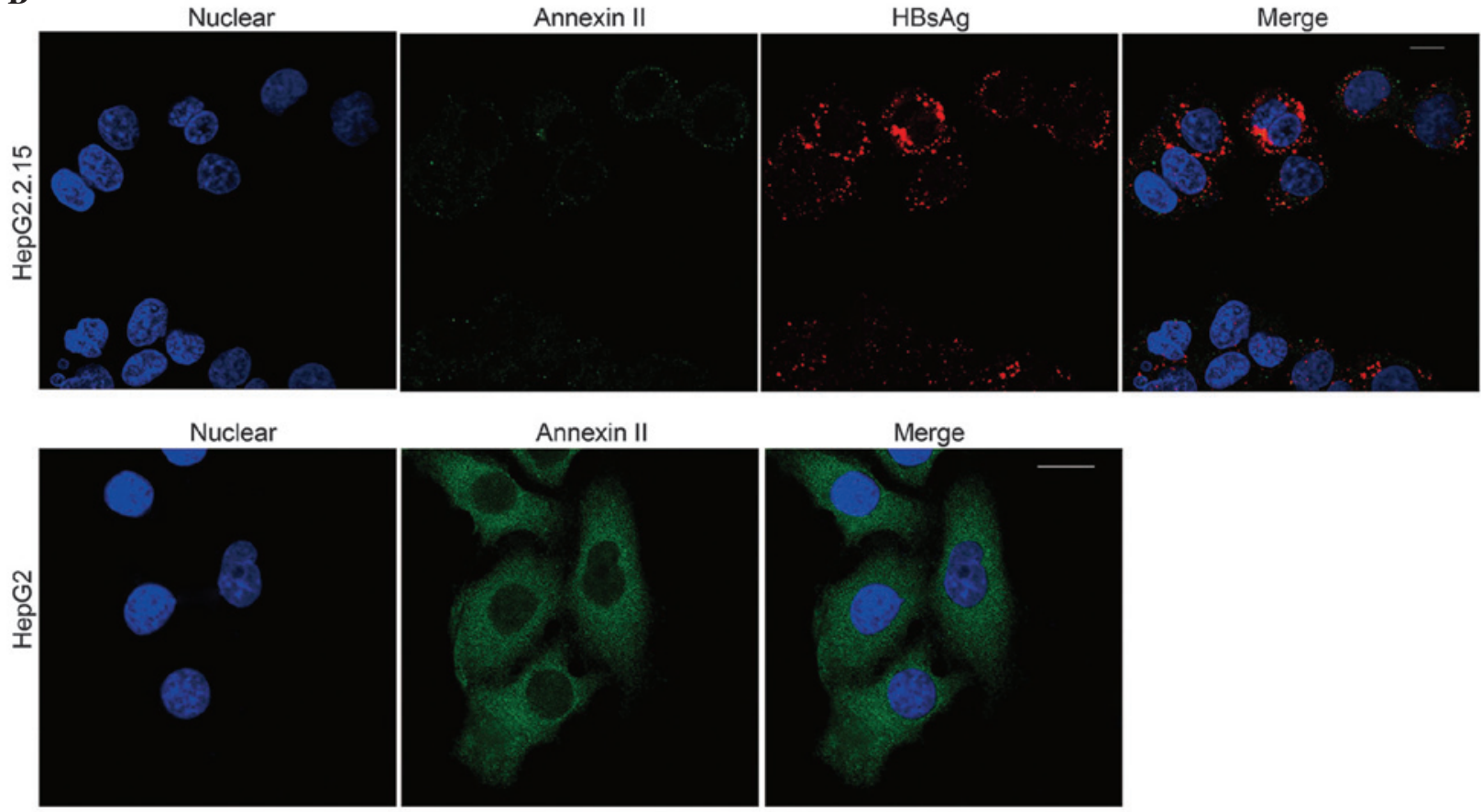

Figure 1. Expression and localization of annexin II in different cells. (A) Intracellular expression of annexin II was determined by western blot analysis in SMMC-7721, HepG2, HepG2.2.15, 293T and CHO cells. CHO cells transfected with the pcDNA3.1-annexin II plasmid were used as a positive control. The level of annexin II was extremely low in the HepG2.2.15 cells, but high in the HepG2 and SMMC-7721 cells. (B) HepG2 and HepG2.2.15 cells were plated onto glass coverslips and incubated for $24 \mathrm{~h}$, then fixed, permeabilized and stained with antibodies against hepatitis B surface antigens (red) and annexin II (green). The cell nuclei were stained blue using Hoechst 33258. Slides were visualized using a laser scanning confocal microscope. Annexin II was distributed diffusely in the cytoplasm and nuclei of the HepG2 and HepG2.2.15 cells, while the cytoplasm exhibited a significantly brighter signal in the HepG2 cells. Annexin II colocalized with HBsAg in the cytosol of HepG2.2.15 cells. CHO, Chinese hamster ovary; HBsAg, hepatitis B surface antigen; Merge, combined staining.

pH 7.5; $150 \mathrm{mM} \mathrm{NaCl}$ and 0.5\% NP-40), supplemented with Roche protease inhibitor-cocktail (Roche Diagnostics, Basel, Switzerland). The samples were sonicated at $15 \%$ power for $60 \mathrm{sec}$ with a $3 \mathrm{sec}$ break every $3 \mathrm{sec}$ and then centrifuged to obtain a clear supernatant. Western blot analysis was performed with the anti-annexin II and anti- $\beta$-actin antibodies to detect the expression of annexin II and $\beta$-actin. The HBV viral antigen HBsAg was examined with $75 \mu$ l supernatant using an Abbott chemiluminescence immunoassay at the Department of Laboratory Medicine, First Hospital of Jilin University.

Statistical analysis. All experiments were repeated 3-5 times with duplicate or triplicate samples for each condition, unless otherwise indicated. A result representative of multiple independent experiments is shown in each figure. Error bars reported in all figures represent the standard deviation of the mean. Statistical analyses were performed using analysis of variance. $\mathrm{P}<0.05$ was considered to indicate a statistically significant difference.

\section{Results}

Intracellular expression and localization of annexin II in different cell lines. Cell lysates were analyzed by western blot analysis to confirm the expression of intracellular annexin II in the SMMC-7721, HepG2, HepG2.2.15, 293T and CHO cell lines (Fig. 1A). CHO cells transfected with the annexin II expression vector were used as a positive control. The expression level of annexin II was extremely low in the HepG2.2.15 cells, minimal in the CHO cells, but high in the HepG2, SMMC-7721 and 293T cells.

Further investigation determined the localization of annexin II in HepG2 and HepG2.2.15 cells by indirect immunofluorescence. The distribution of annexin II was homogenous throughout the cytoplasm and nuclei of the HepG2 and HepG2.2.15 cells, with lower immunoreactivity within the nuclei. The cytoplasm of the HepG2 cells exhibited a markedly higher annexin II signal. Colocalization of annexin II and HBsAg was observed in the cytosol of the HepG2.2.15 cells. (Fig. 1B). 

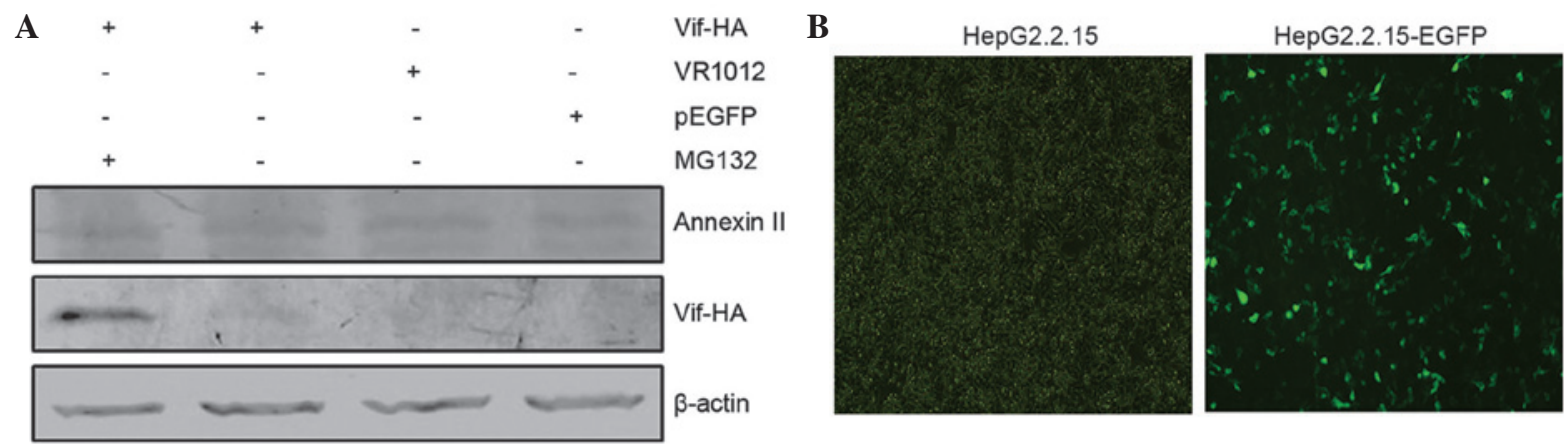

Figure 2. Correlation of the expression of annexin II with ubiquitination and degradation in HepG2.2.15 cells. HepG2.2.15 cells were transfected with the HIV-1 Vif-HA plasmid, control vector (VR1012) or pEGFP-N1 expression plasmid. At 24 h post-transfection, the proteasome inhibitor MG132 was added to one of the Vif-complemented HepG2.2.15 cells. HIV-1 Vif-HA was a positive control for ubiquitination and degradation. (A) Following treatment with MG132 for $16 \mathrm{~h}$, the cells were all lysed and measured by immunoblotting using anti-annexin II and anti-HA antibodies to analyze the expression of annexin II and Vif, respectively. $\beta$-actin was used as an internal control. (B) Transfection efficiency of transfected cells. These data demonstrated that MG132 treatment did not increase the stability of annexin II in the HepG2.2.15 cells. HIV-1, human immunodeficiency virus-type 1; Vif-HA, viral infectivity factor-hemagglutinin; anti-HA, anti-hemagglutinin.

A

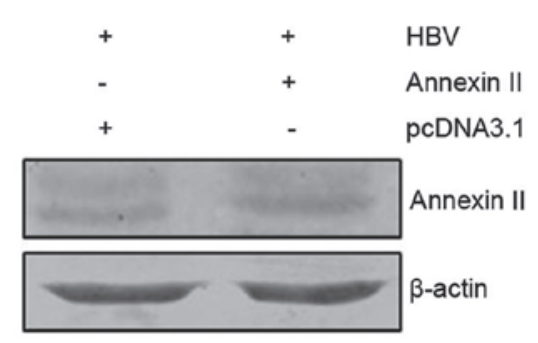

B

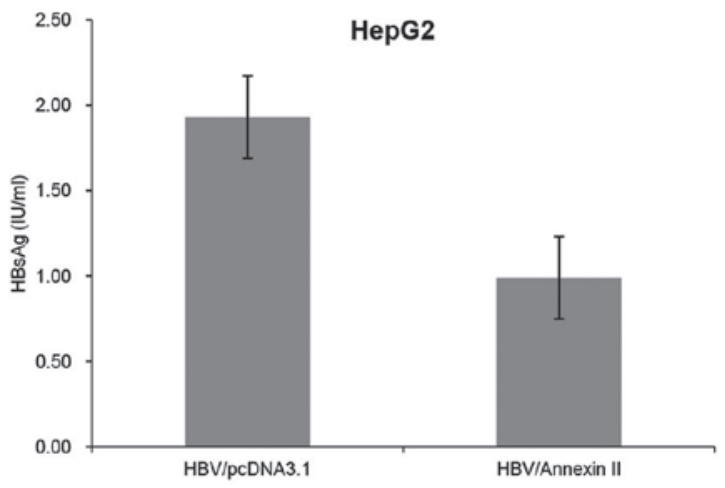

Figure 3. Annexin II mediates the secretion of HBsAg in HBV formation. HepG2 cells were cotransfected with a HBV expression vector and an annexin II plasmid or a control vector (pcDNA3.1). (A) Western blot analysis was performed to detect the expression of annexin II. $\beta$-actin was used for normalization of sample loading. An Abbott chemiluminescence immunoassay was used to quantify the HBsAg titer in the supernatants of transfected HepG2 cells. (B) HBsAg titer was significantly decreased in the cells cotransfected with the $\mathrm{HBV}$ expression vector and annexin II plasmid (analysis of variance; $\mathrm{P}<0.05$, as compared with the control cells cotransfected with HBV expression vector and pcDNA3.1 control vector). HBsAg, hepatitis B surface antigen; HBV, hepatitis B virus.

Ubiquitination and proteasome-mediated degradation of annexin II in HepG2.2.15 cells. The proteasome inhibitor MG132 was added to Vif-complemented HepG2.2.15 cells and HIV-1 Vif was used as a positive control for promoting the polyubiquitination and degradation of substrates (19). The results demonstrated that downregulation of annexin II in the HepG2.2.15 cells was not associated with ubiquitination or potential degradation (Fig. 2). Transfection efficiency in the GFP-complemented HepG2.2.15 cells was observed using a fluorescence microscope (Olympus Corporation, Tokyo, Japan).

Annexin II mediates the secretion of HBsAg in $H B V$ formation. The HepG2 cells were cotransfected with the HBV expression vector and the annexin II plasmid or a control vector. The expression of annexin II was analyzed by western blot analysis in the lysed cotransfected HepG2 cells and $\beta$-actin was used as an internal control (Fig. 3A). An Abbott chemiluminescence immunoassay was used to assess the secretion of HBsAg in the cell culture supernatants. When compared with the control-transfected cells, the HBsAg titer was decreased significantly in the HepG2 cells cotransfected with the HBV expression vector and the annexin II plasmid $(\mathrm{P}<0.05$; Fig. 3B).

\section{Discussion}

The present study demonstrated that the protein expression level of annexin II was lower in the HepG2.2.15 cells compared with the non-HBV-producing hepatoma cells, including HepG2 and SMMC-7721. The cytoplasm of the HepG2 cells also exhibited a significantly brighter signal of annexin II when compared with the HepG2.2.15 cells. In addition, annexin II colocalized with HBsAg in the cytosol of the HepG2.2.15 cells. Previous studies have indicated that annexin II is increased at the mRNA and protein levels in HCC tissues and that the expression of annexin II is associated with the histological grades of $\operatorname{HCC}(6,12,13)$. However, it has been observed that the expression level of annexin II is decreased in HepG2.2.15 cells compared with HepG2 cells $(14,20)$, which is in accordance with the present study. HepG2.2.15 cells are a derivative of HepG2 stably expressing HBV, thus it is hypothesized that the downregulation of annexin II in HepG2.2.15 cells may be attributed to various decisive factors, 
including HBV DNA integration, cytoskeletal rearrangement and proteasome-dependent degradation.

It has been proposed that the low expression of annexin II in HepG2.2.15 cells may be inhibited by the expression of the HBV genome due to DNA methylation in the annexin II promoter (14). However, certain studies have demonstrated that membrane-binding and secreted proteins are targeted by the ubiquitin-proteasome system and thus are degraded (15). HIV-1 Vif was used as a positive control owing to the formation of a E3 ubiquitin ligase with CUL5 and elongin B/C, promoting the polyubiquitination and degradation of various substrates (19). In the present study, the proteasome inhibitor MG132 was added to Vif-complemented HepG2.2.15 cells and no change in the expression of annexin II was demonstrated. These results revealed that downregulation of annexin II in HepG2.2.15 cells was not associated with polyubiquitination or degradation. Overall, the present study confirmed that HBV directly downregulated the expression of annexin II in HepG2.2.15 cells.

In addition, the present study then examined whether annexin II was important in the viral life cycle. As annexin II is a $\mathrm{Ca}^{2+}$-regulated phospholipid-binding and membrane-binding protein, it was hypothesized that annexin II may be associated with viral entry and budding (21-23). Viral entry remains enigmatic, however, envelopment and budding of the mature nucleocapsid is dependent on the presence of viral surface proteins. In addition, the intracellular accumulation of $\mathrm{HBsAg}$ is closely associated with hepatocarcinogenesis (3), which initiates ER stress and induces DNA damage and genomic instability $(5,24)$. The present study found that annexin II colocalized with HBsAg in the cytosol of the HepG2.2.15 cells. Therefore, further investigation was performed to determine whether annexin II affected the secretion of HBsAg for intracellular accumulation. The results demonstrated that annexin II significantly decreased the secretion of HBsAg in the HepG2 cells cotransfected with the HBV and annexin II expression vectors. Taken together, these results confirmed that inhibition of HBsAg secretion by annexin II contributed to intracellular storage of HBsAg and may be an indispensable factor in hepatocarcinogenesis.

In conclusion, HBV decreased the expression of annexin II in HepG2.2.15 cells, however, annexin II significantly inhibited the secretion of HBsAg in favor of intracellular accumulation of HBsAg. These findings provide new insights into the mechanism underlying HBV-induced liver cancer and may assist in the development of novel therapeutic strategies for patients with $\mathrm{HBV}$-associated $\mathrm{HCC}$.

\section{Acknowledgements}

The authors would like to thank the Institute of Virology and AIDS Research and Hepatology Institute, First Hospital of Jilin University, People's Republic of China. The authors would like to thank Dr Carl E. Creutz, Dr Shengqi Wang, Dr Robert J. Schneider and Dr Xiao-Fang Yu for providing the pEGFP-AnxII plasmid, HepG2.2.15 cell line, HBV expression vector and HIV-1 Vif-HA plasmid, respectively. This study was supported in part by funding from the National Natural Science Foundation of China (grant no. 30971353) and the Project of Industrial Technology Research and Development, Jilin province (grant no. 3J1106593428).

\section{References}

1. Kourtis AP, Bulterys M, Hu DJ and Jamieson DJ: HIV-HBV coinfection - a global challenge. N Engl J Med 366: 1749-1752, 2012.

2. Arzumanyan A, Reis HM and Feitelson MA: Pathogenic mechanisms in HBV- and HCV-associated hepatocellular carcinoma. Nat Rev Cancer 13: 123-135, 2013.

3. Chemin I and Zoulim F: Hepatitis B virus induced hepatocellular carcinoma. Cancer Lett 286: 52-59, 2009.

4. Wang HC, Huang W, Lai MD and Su IJ: Hepatitis B virus pre-S mutants, endoplasmic reticulum stress and hepatocarcinogenesis. Cancer Sci 97: 683-688, 2006.

5. Hsieh YH, Su IJ, Wang HC, et al: Pre-S mutant surface antigens in chronic hepatitis B virus infection induce oxidative stress and DNA damage. Carcinogenesis 25: 2023-2032, 2004.

6. Longerich T, Haller MT, Mogler C, et al: Annexin A2 as a differential diagnostic marker of hepatocellular tumors. Pathol Res Pract 207: 8-14, 2011.

7. Paciucci R, Torà M, Díaz VM and Real FX: The plasminogen activator system in pancreas cancer: role of t-PA in the invasive potential in vitro. Oncogene 16: 625-633, 1998.

8. Singh P: Role of Annexin-II in GI cancers: interaction with gastrins/progastrins. Cancer Lett 252: 19-35, 2007.

9. Tomonaga T, Matsushita K, Yamaguchi S, et al: Identification of altered protein expression and post-translational modifications in primary colorectal cancer by using agarose two-dimensional gel electrophoresis. Clin Cancer Res 10: 2007-2014, 2004.

10. Chetcuti A, Margan SH, Russell P, et al: Loss of annexin II heavy and light chains in prostate cancer and its precursors. Cancer Res 61: 6331-6334, 2001.

11. Pena-Alonso E, Rodrigo JP, Parra IC, et al: Annexin A2 localizes to the basal epithelial layer and is down-regulated in dysplasia and head and neck squamous cell carcinoma. Cancer Lett 263: 89-98, 2008.

12. Mohammad HS, Kurokohchi K, Yoneyama H, et al: Annexin A2 expression and phosphorylation are upregulated in hepatocellular carcinoma. Int J Oncol 33: 1157-1163, 2008.

13. Yoon SY, Kim JM, Oh JH, et al: Gene expression profiling of human HBV- and/or HCV-associated hepatocellular carcinoma cells using expressed sequence tags. Int J Oncol 29: 315-327, 2006.

14. Niu D, Sui J, Zhang J, et al: iTRAQ-coupled 2-D LC-MS/MS analysis of protein profile associated with HBV-modulated DNA methylation. Proteomics 9: 3856-3868, 2009.

15. Ciechanover A: The ubiquitin-proteasome pathway: on protein death and cell life. EMBO J 17: 7151-7160, 1998.

16. Glickman MH and Ciechanover A: The ubiquitin-proteasome proteolytic pathway: destruction for the sake of construction. Physiol Rev 82: 373-428, 2002.

17. Eberhard DA, Karns LR, VandenBerg SR and Creutz CE: Control of the nuclear-cytoplasmic partitioning of Annexin II by a nuclear export signal and by p11 binding. J Cell Sci 114: 3155-3166, 2001.

18. Jing X, Piao YF, Liu Y and Gao PJ: Beta2-GPI: a novel factor in the development of hepatocellular carcinoma. J Cancer Res Clin Oncol 136: 1671-1680, 2010.

19. Liu B, Sarkis PT, Luo K, et al: Regulation of Apobec3F and human immunodeficiency virus type 1 Vif by Vif-Cul5-ElonB/C E3 ubiquitin ligase. J Virol 79: 9579-9587, 2005.

20. Niu D, Feng $\mathrm{H}$ and Chen WN: Proteomic analysis of HBV-associated HCC: insights on mechanisms of disease onset and biomarker discovery. J Proteomics 73: 1283-1290, 2010.

21. Laohavisit A and Davies JM: Annexins. New Phytol 189: 40-53, 2011.

22. Hayes MJ, Rescher U, Gerke V and Moss SE: Annexin-actin interactions. Traffic 5: 571-576, 2004.

23. Rescher U and Gerke V: S100A10/p11: family, friends and functions. Pflugers Arch 455: 575-582, 2008.

24. Yeung P, Wong DK, Lai CL, et al: Association of hepatitis B virus pre-S deletions with the development of hepatocellular carcinoma in chronic hepatitis B. J Infect Dis 203: 646-654, 2011. 